Gynäkologe 2017 · 50:176

DOI 10.1007/s00129-016-4002-2

Online publiziert: 13 . Dezember 2016

C) Springer Medizin Verlag Berlin 2016

CrossMark

Das Interesse an der Urogynäkologie hat in den letzten 10 Jahren stark zugenommen. Dies gilt primär für die betroffenen Frauen, aber auch für uns behandelnden Ärzte. Gründe sind dafür zum einen die steigende Lebenserwartung, der zunehmende Anspruch auf Lebensqualität in jeder Altersgruppe und Enttabuisierungen: der Beckenbodenfunktionsstörungen im Allgemeinen und der Inkontinenzproblematik im Speziellen. Zum anderen haben sich in den vergangenen Jahren die Behandlungsoptionen und damit die Erfolgsaussichten auf Heilung oder zumindest relevante Besserung und damit auf den Erhalt bzw. die Wiederherstellung der Lebensqualität erheblich verbessert.

Neben allen technischen Innovationen ist einer der wesentlichen Gründe dafür die Entwicklung hochkarätiger Leitlinien durch die Arbeitsgemeinschaft für Urogynäkologie und plastische Beckenbodenrekonstruktion (AGUB), eine der größten Arbeitsgemeinschaften der Deutschen Gesellschaft für Gynäkologie und Geburtshilfe (DGGG) mit über 1000 Mitgliedern. Diese erfolgte gemeinsam mit den Kollegen aus Österreich und der Schweiz und deckt alle wesentlichen urogynäkologischen Krankheitsbilder ab.

Daneben haben wir Strukturen zur persönlichen und institutionellen Qualifizierung und Zertifizierung etabliert: Die persönliche Qualifizierung AGUB(Arbeitsgemeinschaft für Urogynäkologie und plastische Beckenbodenrekonstruktion)-I-III in Analogie zur DEGUM(Deutsche Gesellschaft für Ultraschall in der Medizin)-Zertifizierung und die Zertifizierung der strukturellen/ institutionellen Voraussetzungen über die 3 zentralen an der Behandlung dieser Krankheitsbilder beteiligten Fachgesellschaften, der Deutschen Gesellschaft für Allgemein- und Viszeralchirurgie, der

\title{
Thomas Dimpfl
}

Frauenklinik, Klinikum Kassel, Kassel, Deutschland

\section{Urogynäkologie}

Deutschen Gesellschaft für Koloproktologie, der Deutschen Gesellschaft für Urologie und der Deutschen Gesellschaft für Gynäkologie und Geburtshilfe.

Durch den Ausbau der vorhandenen Strukturen der Weiterbildung und die permanente Verbesserung der persönlichen Qualifikationen der in Klinik und Praxis tätigen Kollegen wird die lange urogynäkologische Tradition unseres Faches hervorragend fortgesetzt. Der steigende Qualitätsanspruch und die Vielseitigkeit der Beckenbodenprobleme erfordern die Implementierung der Subspezialität Urogynäkologie, wie im europäischen Modell vorgegeben. An deren Umsetzung wird aktuell intensiv gearbeitet.

》) An der unabdingbaren
Implementierung der
Subspezialität Urogynäkologie
wird intensiv gearbeitet

Im dem Ihnen vorliegenden Heft mit dem Themenschwerpunkt Urogynäkologie werden Ihnen zur Vertiefung des Wissens wesentliche Aspekte der Subspezialität Urogynäkologie von namhaften Vertretern dargestellt und diskutiert.

Gert Naumann stellt eingangs die urogynäkologische Diagnostik als Grundlage jeder erfolgreichen Behandlung dar. Eine sinnvolle und pragmatische Diagnostik mit individueller Nutzung von Urodynamik und Beckenbodensonographie ist Grundpfeiler für den Start einer abgestuften Therapie aller Beckenbodenfunktionsstörungen. Ursula Peschers zeigt die konservativen und operativen Möglichkeiten der Behandlung der Dranginkontinenz auf. Dabei liegt der Fokus auf der Problematik der Polypharmazie bei multimorbiden
Patientinnen und der kritischen Beurteilung relativ neuer Verfahren wie der Botulinumtoxininjektion und der sakralen Neuromodulation. Ein standardisiertes perioperatives Management hilft, Komplikationen $\mathrm{zu}$ vermeiden bzw. rechtzeitig zu erkennen. Ralf Tunn mit Mitarbeitern stellt vor, wie profitieren Patientinnen und medizinisches Personal davon gleichermaßen profitieren. Christl Reisenauer et al. fassen in ihrem Artikel die wichtigsten Krankheitsbilder aus dem koloproktologischen Fachbereich zusammen. Proktologische Erkrankungen und Symptome werden häufig im Rahmen der gynäkologischen Untersuchung erstmalig benannt. Darüber hinaus berichtet ein Großteil unserer Patientinnen über kombinierte Beschwerden, sodass eine erfolgversprechende Behandlung nur über eine enge Kooperation mit den Koloproktologen möglich ist. Abschließend stellen wir die aktuellen Behandlungsmöglichkeiten bei Descensus genitalis zusammen.

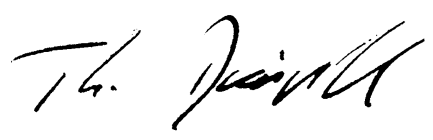

Thomas Dimpfl mit den Autoren

\section{Korrespondenzadresse}

Prof. Dr. T. Dimpfl

Frauenklinik, Klinikum Kassel

Mönchebergstr. 41-43, 34125 Kassel,

Deutschland

dimpfl@klinikum-kassel.de

Interessenkonflikt. T. Dimpfl gibt an, dass kein Interessenkonflikt besteht. 